EPJ Web of Conferences 41, 11001 (2013)

DOI: $10.1051 /$ epjconf/20134111001

C) Owned by the authors, published by EDP Sciences, 2013

\title{
Spatial and Spectral Coherent Control over Direct Frequency Comb Excitation
}

\author{
I. Barmes ${ }^{1}$, S. Witte ${ }^{1}$, and K.S.E Eikema ${ }^{1}$ \\ ${ }^{1}$ LaserLaB Amsterdam, VU University, De Boelelaan 1081, 1081HV Amsterdam, The Netherlands
}

\begin{abstract}
We demonstrate a method for spatial control over two-photon excitation, by using counter-propagating frequency comb pulse trains. This type of control is shown to enable significant improvement for direct frequency comb spectroscopy.
\end{abstract}

\section{Introduction}

The ability to control quantum processes is a major goal in physics, chemistry and biology. The outcome of a quantum process is often dependent on interference between many different pathways to the same final state. This gives unique possibilities for coherent quantum control, but also provides a challenge as the system needs to be manipulated in a phase-coherent way. Various demonstrations of coherent control have been reported in a wide range of topics, including atomic physics [1], chemical reactions [2] and light-driven biological processes [3]. Many of these studies have focused on the spectral amplitude and phase manipulation of single ultrafast laser pulses. In high-resolution spectroscopy, the use of the phase coherence between many consecutive pulses from a modelocked oscillator has led to the advent of frequency combs [4,5]. The stabilization of the pulse-to-pulse carrier-envelope phase slip and the oscillator repetition rate provides a comb of equally spaced optical modes with precisely determined absolute frequencies.

By combining the techniques of single-pulse coherent control with the long-term coherence of frequency combs, a versatile and highly accurate new type of quantum control can be obtained. In addition, by using two counter-propagating laser beams instead of a single beam, we can exploit new degrees of freedom and achieve spatially resolved control over two-photon excitation. We demonstrate the capabilities of this new approach by measuring the 5S-7S two-photon transition in rubidium with unprecedented accuracy, using a compact and robust setup. Through the use of coherent control techniques, we can effectively minimize detrimental decoherence effects such as Doppler broadening, which traditionally plague high-resolution measurements [6].

The signal produced by counter-propagating pulse trains has two contributions. The first contribution arises due to excitation from co-propagating photon pairs from either from the left or the right side. This contribution is spatially independent and is Doppler-broadened due to the large velocity spread of the atoms in a room temperature gas cell [1]. In the second contribution the photons of each pair come from opposite directions. This contribution reduces the Doppler width of the excitation and is spatially dependent. The signal due to the second contribution can be written as:

$$
S_{2 p}(z)=\int_{-\infty}^{\infty} d \omega^{\prime} \int_{-\infty}^{\infty} d \omega^{\prime \prime} A\left(\omega^{\prime}\right) A\left(-\omega^{\prime}\right) A\left(\omega^{\prime \prime}\right) A\left(-\omega^{\prime \prime}\right) e^{i\left(\Phi\left(\omega^{\prime}\right)+\Phi\left(-\omega^{\prime}\right)-\Phi\left(\omega^{\prime \prime}\right)-\Phi\left(-\omega^{\prime \prime}\right)\right)} e^{i \frac{2 z}{c}\left(\omega^{\prime}-\omega^{\prime \prime}\right)}
$$


where the frequency $\omega$ is defined relative to half the transition frequency $\omega_{0} / 2$, and $z$ is the spatial coordinate along the laser propagation axis. From this equation, we find that an anti-symmetric phase mask (with respect to the transition frequency) does not affect the excitation probability, and leads to the same signal as one would acquire with transform-limited pulses. This behavior is similar to the case of single-sided excitation [1]. A new level of control is made possible by applying symmetric spectral phase masks, which leads to the generation of complex spatial excitation patterns. These patterns can extend over large regions and can be manipulated with great flexibility. Interestingly, integrating Eq.1 over $z$ leads to an expression in which all phase terms cancel, with the result that the total excitation rate is independent of the spectral phase. This property can be exploited for new coherent control schemes, as the spatial manipulation of the signal does not degrade the total signal strength.

\section{Setup for spatial control}

We employ a modelocked femtosecond Ti:sapphire oscillator, with its repetition frequency and carrier-envelope phase-offset stabilized to a GPS-disciplined Rb atomic clock. Spectral phase control is implemented through a 640-pixel phase-only spatial light modulator placed at the Fourier plane of a standard $4 \mathrm{f}$ system. The interaction setup consists of a $\mathrm{Rb}$ atomic vapor cell heated to $\sim 60^{\circ} \mathrm{C}$. The laser beam is focused in the vapor cell, after which it is back-reflected such that two consecutive frequency comb pulses overlap in the center of the $\mathrm{Rb}$ cell. The transition is observed by detecting cascade fluorescence at $420 \mathrm{~nm}$ through the $6 \mathrm{P}$ level, using either an EMCCD camera or a photomultiplier tube. An illustration of the interaction setup is given in Fig. $1 \mathrm{~A}$ and $1 \mathrm{C}$, and the resulting excitation patterns are shown in Fig. 1B and 1D.
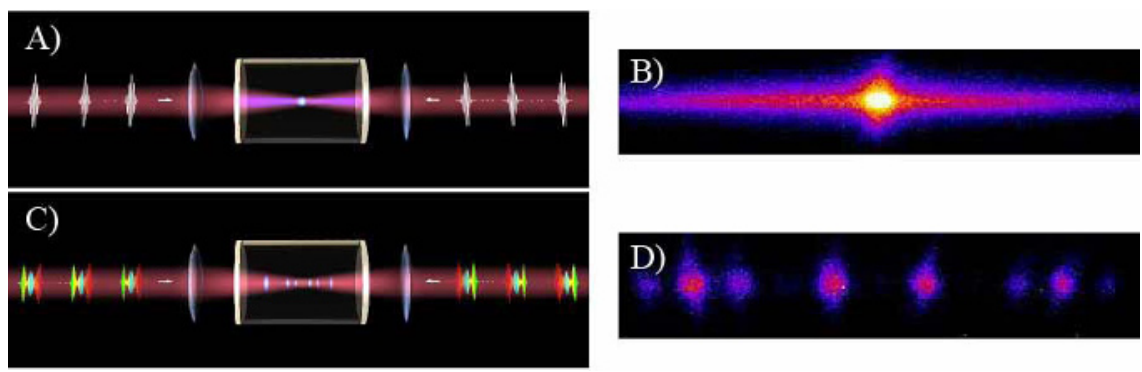

Fig. 1. Schematic principle of spatial coherent control in a counter-propagating pulse geometry. A) Excitation in a gas cell using transform-limited pulses. B) Measured spatial excitation pattern along the laser propagation direction, for transform-limited pulses. A bright spot occurs where the counterpropagating pulses overlap, while the single-sided excitation appears as a long, bright stripe. C) Excitation with spectrally shaped counter-propagating pulses. D) A cosine-shaped spectral phase allows the generation of complex spatial excitation patterns.

\section{Results}

Spatial coherent control can be used to overcome an important limitation in high-precision twophoton spectroscopy with frequency comb pulses. In traditional two-photon spectroscopy two counter-propagating CW laser beams are used to minimize Doppler broadening in a room temperature gas sample. This method breaks down when using ultrashort pulses from a frequency comb laser, because the overlap region of these pulses is extremely small. The single-sided excitation (in this case Doppler-broadened background) degrades the signal-to-noise ratio and hampers high-precision spectroscopy. Spatial coherent control provides a way to eliminate the background, while control over the absolute frequencies of the frequency comb enables scanning over the desired transition. 
We demonstrate this method by performing direct frequency comb spectroscopy on the four allowed 5S-7S two-photon transitions in atomic rubidium. As the goal of spatial coherent control in this case is only to reduce the single-sided excitation, the exact spatial pattern is not important. We therefore employ a particularly simple spectral phase mask that not only completely eliminates the background but is also very easy to realize experimentally. The SLM in the Fourier plane is replaced by two mirrors, each reflecting half of the spectrum around $\omega_{0} / 2$. By translating one of the mirrors a time delay is induced between the "red" and the "blue" parts of the spectrum leading to a complete elimination of the Doppler-broadened background. The effect of this spectral phase is shown in Fig. $2 \mathrm{~A}$ and $2 \mathrm{~B}$. We scan the absolute position of the frequency comb modes across the four allowed transitions. Such a scan is shown in Fig. $2 \mathrm{C}$ where the improved signal-to-noise ratio is clearly visible.

We performed measurements of the ${ }^{85} \mathrm{Rb}(\mathrm{F}=3-3)$ transition at various experimental conditions to account for all possible systematic effects. We found an absolute frequency of 788,795,814,061 (20) $\mathrm{kHz}$, improving the literature value by a factor 2 . In addition, we have measured the hyperfine structure and isotope shift of the $\mathrm{Rb} 5 \mathrm{~S}-7 \mathrm{~S}$ transition with an improvement in accuracy of up to an order of magnitude. The introduction of quantum control now paves the way for applying direct frequency comb spectroscopy to a much wider range of systems.

\section{Conclusion}

We find that the use of coherent control methods in a counter-propagating geometry provides exciting new possibilities for quantum control, giving rise to spatially resolved excitation as well as high-resolution direct frequency comb spectroscopy. We plan to use this principle to improve XUV direct frequency comb spectroscopy [7], enabling accurate tests of quantum electrodynamics.
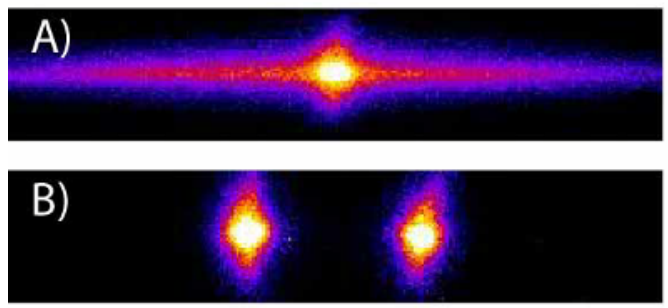

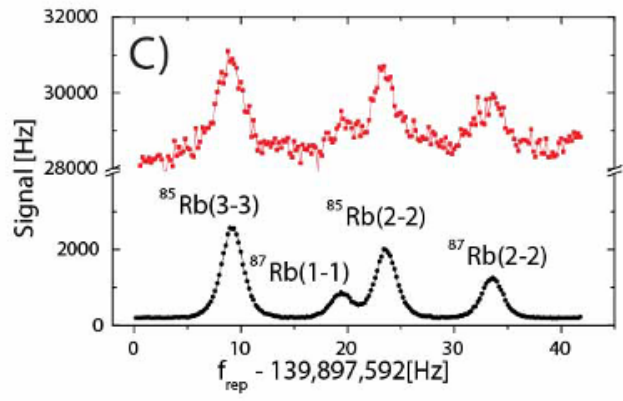

Fig. 2. A) Spatial excitation profile with transform-limited pulses, where the signal from the counterpropagating pulses sits on top of a large, Doppler-broadened background. B) Spatial excitation profile when applying a $\mathrm{V}$-shaped spectral phase to the pulses, which turns off the single-sided excitation. C) Direct frequency comb spectroscopy of the 5S-7S transition in Rb for transform-limited (red) and spatially controlled (black) pulse trains.

\section{References}

1. D. Meschulach, Y. Silberberg, Nature 396, 239 (1998)

2. A. Assion et al., Science 282, 919 (1998).

3. J.L. Herek et al., Nature 417, 533 (2002).

4. R. Holzwarth et al., Phys. Rev. Lett. 85, 2264 (2000).

5. D.J. Jones et al., Science 288, 635 (2000).

6. S. Witte et al., Science 307, 400 (2005).

7. D.Z. Kandula et al., Phys. Rev. Lett. 105, 063001 (2010). 